\title{
The ranks of central factor and commutator groups
}

\author{
Leonid A. Kurdachenko and Pavel Shumyatsky
}

\begin{abstract}
The Schur Theorem says that if $G$ is a group whose center $Z(G)$ has finite index $n$, then the order of the derived group $G^{\prime}$ is finite and bounded by a number depending only on $n$. In the present paper we show that if $G$ is a finite group such that $G / Z(G)$ has rank $r$, then the rank of $G^{\prime}$ is $r$-bounded. We also show that a similar result holds for a large class of infinite groups.
\end{abstract}

The famous Schur Theorem says that if $G$ is a group whose center $Z(G)$ has finite index $n$, then the order of the derived group $G^{\prime}$ is finite and bounded by a number depending only on $n$. Throughout the paper we say that a quantity is $\{a, b, c, \ldots\}$-bounded if it is bounded by a number that depends only on the parameters $a, b, c, \ldots$ The theorem provides a very useful tool for group theorists. Quite naturally, ever since the theorem was proved the relation between $G / Z(G)$ and $G^{\prime}$ was in the focus of considerable attention. One rather straightforward generalization of the Schur theorem is that if $G / Z(G)$ is locally finite, then $G^{\prime}$ is locally finite as well (see [11, p. 102] for example). On the other hand, there is a well-known example, due to Adian, of a torsionfree group $G$ such that $G / Z(G)$ is periodic and even has prime exponent [1]. Thus, periodicity of $G / Z(G)$ in general does not imply that of $G^{\prime}$. Using the positive solution to the restricted Burnside problem [14, 15. A. Mann showed that if $G / Z(G)$ is locally finite and has finite exponent $n$, then $G^{\prime}$ is locally finite and has finite $n$-bounded exponent [8]. A number of other results in the spirit of the Schur theorem can be found in $[10,7,4]$. In the present paper we examine the situation where $G / Z(G)$ has finite rank. Recall that a group $K$ has finite rank $r$ if every finitely generated subgroup of $K$ can be generated by at most $r$

1991 Mathematics Subject Classification. 20D25, $20 \mathrm{~F} 14$.

Key words and phrases. groups of finite rank, the Schur theorem.

The work of the second author was supported by CNPq-Brazil. 
elements. We would like to thank A. Yu. Olshanskii for explaining to us how it can be shown that there exists a group $G$ with the property that $Z(G)$ is a free abelian group of infinite rank while $G$ is a perfect group such that all proper subgroups of $G / Z(G)$ are of prime order. The proof of this fact goes as follows. In Section 27 of [9] one finds the construction of the group $G(\infty)$ as a limit of the sequence $\{G(i)\}$. All proper subgroups of $G(\infty)$ have prime order [9, Theorem 28.1]. The combination of Lemma 27.2, Lemma 25.1 and Theorem 31.1(2) of [9] guarantees that for the aspherical corepresentation $G(\infty)=F / N$ the group $N /[N, F]$ is a free abelian group of infinite rank. Now arguing as in the proof of Corollary 31.2 we deduce that the Schur multiplier of $G(\infty)$ is a free abelian group of infinite rank. The proof also uses the fact that the group $G(\infty)$ does not coincide with $G(i)$ for any $i=1,2, \ldots$. This can be shown by arguing as in the proof of Theorem 19.3 and replacing the reference to Theorem 19.1 by that to Theorem 26.2 .

Thus, finiteness of the rank of $G / Z(G)$ in general does not imply that of $G^{\prime}$. We will show however that under some reasonable additional hypothesis on the group $G$ we do have a rank version of the Schur theorem.

Our first result provides a rank version of the Schur theorem for finite groups.

TheOrem 1. Let $G$ be a finite group such that $G / Z(G)$ has rank $r$. Then the rank of $G^{\prime}$ is $r$-bounded.

The proof of the above theorem depends on the classification of finite simple groups. The Lubotzky-Mann theory of powerful $p$-groups [2] plays an important role in the proof as well. From Theorem 1 we deduce a rank version of the Schur theorem for a large class of infinite groups.

A group $G$ is called generalized radical if it has an ascending series whose quotients are either locally nilpotent or locally finite. Accordingly, a group $G$ is locally generalized radical if every finitely generated subgroup of $G$ is generalized radical.

THEOREM 2. Let $G$ be a locally generalized radical group such that $G / Z(G)$ has finite rank $r$. Then the rank of $G^{\prime}$ is finite and $r$-bounded.

In the present paper we make no attempts to write down explicit bounds for the rank of $G^{\prime}$ in Theorems 1 and 2. Throughout the paper we use without explicit references the facts that if $r(G)=r$, then every subgroup and every quotient of $G$ has rank at most $r$ and that if $G$ 
has a normal subgroup $N$ such that $r(N)=r_{1}$ and $r(G / N)=r_{2}$, then $r(G) \leq r_{1}+r_{2}$.

The next lemma is well-known but for the reader's convenience we include the proof.

LEMMA 3. Let $G$ be a group having a subset $X$ and a normal abelian subgroup $N$ such that $G=\langle N, X\rangle$. Then $[N, G]=\prod_{x \in X}[N, x]$.

Proof. Set $K=\prod_{x \in X}[N, x]$. It is clear that $K \leq[N, G]$ so we need to prove the other inclusion. If $K=1$, then $N$ is central in $G$ and therefore $[N, G]=1$. Thus, it is sufficient to show that $K$ is normal in $G$. The subgroup $N$ normalizes $K$ because $N$ is abelian and $K \leq N$. Therefore we only need to show that every element $x$ of $X$ normalizes $K$. It is straightforward that $x$ normalizes every subgroup of $N$ that contains $[N, x]$. Since $[N, x] \leq K$ for all $x \in X$, the lemma follows.

LEMMA 4. Let $G$ be a group such that $G / Z(G)$ has finite rank $r$. Let $M$ be a normal abelian subgroup of $G$. Then $[M, G]$ has rank at most $r^{2}$.

Proof. Set $N=M Z(G)$ and suppose first that $G$ is finitely generated. Then $N$ is a normal abelian subgroup of $G$ such that $G / N$ can be generated by at most $r$ elements. Therefore we can choose elements $x_{1}, \ldots, x_{r} \in G$ such that $G=\left\langle N, x_{1}, \ldots, x_{r}\right\rangle$. By Lemma 3 $[N, G]=\prod\left[N, x_{i}\right]$. We note that for any $x \in G$ the map that takes every element $y \in N$ to $y^{-1} y^{x}$ is a homomorphism of $N$ in $[N, x]$ whose kernel is $C_{N}(x)$. Since $G / Z(G)$ has rank $r$, it follows that the rank of $N / C_{N}(x)$ is at most $r$ for all $x \in G$ and hence the rank of $[N, x]$ is at most $r$ as well. The equality $[N, G]=\prod\left[N, x_{i}\right]$ now guarantees that the rank of $[N, G]$ is bounded by $r^{2}$. Finally, we remark that $[N, G]=[M, G]$ and so in the case where $G$ is finitely generated the lemma follows.

Let us now drop the assumption that $G$ is finitely generated. Suppose that $[M, G]$ has rank at least $r^{2}+1$. Thus, we can choose elements $y_{1}, \ldots, y_{r^{2}+1} \in[M, G]$ such that the subgroup $\left\langle y_{1}, \ldots, y_{r^{2}+1}\right\rangle$ cannot be generated by $r^{2}$ elements. We can also choose a finitely generated subgroup $K$ in $G$ such that $y_{1}, \ldots, y_{r^{2}+1} \in[M, K] \cap K$. This yields a contradiction since we know that for finitely generated groups the lemma holds. The proof is now complete.

LEMMA 5. Let $d$ and $r$ be positive integers and $G$ a group such that $G / Z(G)$ is soluble with derived length $d$ and $r(G / Z(G))=r$. Then $G^{\prime}$ has finite rank and $r\left(G^{\prime}\right) \leq(1 / 2) d r(r-1)+(d-1) r^{2}$.

Proof. Denote the expression $(1 / 2) d r(r-1)+(d-1) r^{2}$ by $N(d, r)$. If the result is false, then $G^{\prime}$ contains elements $y_{1}, \ldots, y_{N(d, r)+1}$ such 
that the subgroup $\left\langle y_{1}, \ldots, y_{N(d, r)+1}\right\rangle$ cannot be generated by less than $N(d, r)+1$ elements. We can choose a finitely generated subgroup $K$ of $G$ such that $y_{1}, \ldots, y_{N(d, r)+1} \in K^{\prime}$ and so $K$ provides a counterexample to the lemma. Thus, it is sufficient to prove the lemma for finitely generated groups and so we assume that $G$ is finitely generated. It follows that $G / Z(G)$ can be generated by $r$ elements. In particular we deduce that $G=\left\langle Z(G), x_{1}, \ldots, x_{r}\right\rangle$ for suitable $x_{1}, \ldots, x_{r} \in G$.

The lemma will be proved by induction on $d$. Suppose first that $d=1$. Then $G$ is nilpotent of class at most two and, since $G=$ $\left\langle Z(G), x_{1}, \ldots, x_{r}\right\rangle$, it is clear that $G^{\prime}$ is generated by the commutators $\left[x_{i}, x_{j}\right]$, where $1 \leq i<j \leq r$. There are at most $(1 / 2) r(r-1)$ such commutators and so in the case where $d=1$ the lemma holds.

Now we assume that $d \geq 2$ and that the rank of the derived group of $G^{\prime} Z(G)$ is at most $N(d-1, r)$. Of course, the derived group of $G^{\prime} Z(G)$ is precisely $G^{\prime \prime}$, the second derived group of $G$. We pass to the quotient $G / G^{\prime \prime}$ and assume that $G$ is metabelian. Then, by Lemma 4 , the rank of $\left[G^{\prime}, G\right]$ is at most $r^{2}$. The quotient $G /\left[G^{\prime}, G\right]$ is nilpotent of class at most two and so by what we have established above the rank of $G^{\prime} /\left[G^{\prime}, G\right]$ is at most $(1 / 2) r(r-1)$. Hence, in the case where $G$ is metabelian the rank of $G^{\prime}$ is at most $(1 / 2) r(r-1)+r^{2}$. By the induction hypothesis the rank of $G^{\prime \prime}$ is at most $N(d-1, r)$ and so we deduce that the rank of $G^{\prime}$ is at most $(1 / 2) r(r-1)+r^{2}+N(d-1, r)=N(d, r)$, as required.

Lemma 6. There exists an integer $s=s(n, r)$ depending only on $n$ and $r$ such that if $G$ is a finite p-group of exponent dividing $p^{n}$ and rank at most $r$, then the order of $G$ is at most $p^{s}$.

Proof. By Theorem 2.13 of [2] $G$ has a powerful characteristic subgroup $N$ of index at most $p^{\mu(r)}$, where $\mu(r)$ is a number depending only on $r$. Corollary 2.8 in [2] shows that $N$ is a product of at most $r$ cyclic subgroups. Therefore $N$ is of order at most $p^{n r}$ and the lemma follows.

Proposition 7. If $G$ is a finite nilpotent group, then the rank of $G^{\prime}$ is bounded in terms of $r=r(G / Z(G))$ only.

Proof. It is clear that without loss of generality we can assume that $G$ is a $p$-group for some prime $p$. Let $n$ be the least number such that $2^{n} \geq r+2$. Then obviously $G$ does not involve the wreath product $C_{p} \prec C_{p^{n}}$. Let $K=G^{\prime}$ and $H=K^{p^{n}}$ if $p$ is odd and $H=K^{p^{n+1}}$ if $p=2$. By [12] $H$ is powerful. Let $\bar{G}=G / H^{p}$ and $\bar{K}=K / H^{p}$. Obviously $\bar{K}$ has exponent dividing $p^{n+2}$. By the hypothesis $\bar{G} / Z(\bar{G})$ has rank at most $r$ and therefore, by Lemma $6, \bar{K} / Z(\bar{K})$ has order at most $p^{s}$ 
for some $r$-bounded number $s$. Let $d$ denote the derived length of $\bar{G}$. It is clear that $d \leq s+1$ and in particular $d$ is $r$-bounded. Lemma 5 now tells us that the rank, say $t$, of $\bar{K}$ is $r$-bounded. Since $H^{p}$ is the Frattini subgroup of $H$ and since $H / H^{p}$ can be generated by $t$ elements, it follows that $H$ can be generated by $t$ elements. Taking into account that $H$ is powerful, we deduce that $H$ is of rank at most $t$. In particular $H^{p}$ is of rank at most $t$. Combining this with the fact that $\bar{K}$ has rank at most $t$ we now conclude that the rank of $K$ is at most $2 t$, as required.

A well-known theorem of Zassenhaus says that whenever $F$ is a field, the derived length of any soluble subgroup of $G L_{n}(F)$ is bounded by a function of $n$ only $1 \mathbf{1 3}$. This will be used in the following lemma. Given a group $G$, we denote by $F(G)$ the Fitting subgroup of $G$.

LEMma 8. Let $G$ be a finite soluble group of rank $r$. Then the derived length of $G / F(G)$ is r-bounded.

Proof. Consider an unrefinable normal series

$$
G=N_{1}>N_{2}>\cdots>N_{k}>N_{k+1}=1
$$

in $G$. The factors $N_{i} / N_{i+1}$ are elementary abelian of rank at most $r$ and so every factor $N_{i} / N_{i+1}$ can be viewed as a linear space of dimension at most $r$ over some field with $p$ elements. By Zassenhaus' Theorem there is an integer $d$ depending only on $r$ such that every soluble group of automorphisms of $N_{i} / N_{i+1}$ has derived length at most $d$. Let $K$ be the $d$ th derived group of $G$. Then $K$ centralizes every factor of the series

$$
G=N_{1}>N_{2}>\cdots>N_{k}>N_{k+1}=1
$$

and hence $K$ is nilpotent. Thus, $K \leq F(G)$ and $G / F(G)$ has derived length at most $d$.

In the proof of the next lemma we use the well-known corollary of the classification of finite simple groups that Aut $S / \operatorname{Inn} S$, the outer automorphism group of $S$, is soluble for every finite simple group $S$ (this fact is also known under the name of the Schreier Conjecture).

LEMMA 9. Let $G$ be a finite group of rank $r$ and assume that $G$ has no nontrivial normal soluble subgroups. Then $G$ has a normal series

$$
M \leq T \leq G
$$

such that $M$ is isomorphic to a direct product of at most $r$ non-abelian simple groups, $T / M$ is soluble and $G / T$ has order at most $r$ !. 
Proof. Let $M$ be the product of all minimal normal subgroups of $G$. Of course, $M=S_{1} \times S_{2} \times \cdots \times S_{k}$, where the subgroups $S_{1}, S_{2}, \ldots, S_{k}$ are isomorphic with non-abelian simple groups. Since $r(M) \leq r$ and since all subgroups $S_{1}, S_{2}, \ldots, S_{k}$ have even order, it follows that $k \leq r$.

Our group $G$ acts by conjugation on $M$ and this action induces a natural homomorphism of $G$ in the symmetric group on $k$ symbols. Let $T$ be the kernel of the homomorphism. In other words, $T$ is the intersection of the normalizers $N_{G}\left(S_{i}\right)$ for $i=1,2, \ldots, k$. Let $U$ be the last term of the derived series of $T$. Thus, $U$ is the intersection of all normal subgroups $N$ of $T$ such that $T / N$ is soluble. For every $i=$ $1,2, \ldots, k$ put $T_{i}=S_{i} C_{T}\left(S_{i}\right)$. Then $T / T_{i}$ embeds into Aut $S_{i} /$ Inn $S_{i}$, which is soluble. Therefore $U \leq T_{i}$ for all $i=1,2, \ldots, k$. Hence, any element in $U$ induces an inner automorphism of $S_{i}$ for all $i \leq k$. It follows that for every $x \in U$ there exist elements $x_{i} \in S_{i}$ such that

$$
x_{1} x_{2} \ldots x_{k} x^{-1} \in C_{T}\left(S_{i}\right) \text { for all } i=1,2, \ldots, k .
$$

Thus, $x_{1} x_{2} \ldots x_{k} x^{-1} \in C_{T}(M)$. Since $G$ has no nontrivial normal soluble subgroups, it follows that $C_{T}(M)=1$ and therefore $x \in M$. Thus, $U=M$ and $T / M$ is soluble.

Since $G / T$ embeds in the symmetric group on $k$ symbols, it follows that the index of $T$ in $G$ is at most $r$ !. The proof is complete.

Proof of Theorem 1. Recall that $G$ is a finite group such that $G / Z(G)$ has rank $r$. Let $R$ be the maximal normal soluble subgroup of $G$ and $F=F(R)$. Since $R / Z(R)$ has rank at most $r$ and since $F(R / Z(R))=F / Z(R)$, Lemma 8 shows that the derived length of $R / F$ is $r$-bounded. By Proposition $7 F^{\prime}$ has $r$-bounded rank and therefore we can pass to the quotient $G / F^{\prime}$. Hence, without loss of generality we can assume that the derived length of $R$ is $r$-bounded. Now Lemma 5 guarantees that the rank of $R^{\prime}$ is $r$-bounded and we pass to the quotient $G / R^{\prime}$. Thus, we will assume that $R$ is abelian. Since $G / R$ has rank $r$, we can choose $r$ elements $a_{1}, \ldots, a_{r}$ such that $G=\left\langle R, a_{1}, \ldots, a_{r}\right\rangle$. Then, by Lemma 4, the rank of $[R, G]$ is at most $r^{2}$. Passing to the quotient $G /[R, G]$ assume that $R=Z(G)$.

The structure of $G / R$ is described in Lemma 9. We assume that $G \neq R$ and let $M / R$ be the the product of all minimal normal subgroups of $G / R$. Then $M / R$ is a direct product of $k \leq r$ simple nonabelian groups. For $i=1, \ldots, k$ let $S_{i}$ denote the subgroup of $G$ such that $S_{i} / R$ is a simple factor of $M / R$. Of course, $M=S_{1} S_{2} \ldots S_{k}$. Let $D_{i}$ be the derived group of $S_{i}$ for $i=1,2, \ldots, k$. Then $D_{i}$ is a perfect group and $D_{i} / Z\left(D_{i}\right)$ is a simple group of rank at most $r$. Thus, $D_{i}$ is a so-called quasisimple group. The Schur multipliers of all simple groups 
are well-known and can be found in [5, p. 302-303]. All of them are abelian groups of rank at most three. Thus, the rank of $D_{i}$ is at most $r+3$. From this we deduce that the product $D=D_{1} D_{2} \ldots D_{k}$ has rank at most $r(r+3)$. Thus, we pass to the quotient $\bar{G}=G / D$. Lemma 9 tells us that $\bar{G}$ has a normal soluble subgroup $\bar{T}=T / D$ of index at most $r$ !. Precisely in the same way as we have shown above that the rank of $[R, G]$ is $r$-bounded we can now show that the rank of $[\bar{T}, \bar{G}]$ is $r$-bounded as well. Passing to the quotient $\bar{G} /[\bar{T}, \bar{G}]$, assume that $\bar{T}$ is central. Then the index of $Z(\bar{G})$ in $\bar{G}$ is at most $r$ ! and by Schur's theorem the order of the derived group of $\bar{G}$ is $r$-bounded. The proof is now complete.

A routine inverse limit argument along the lines of [6] now shows that if $G$ is a locally finite group such that $G / Z(G)$ has rank $r$, then the rank of $G^{\prime}$ is $r$-bounded. The following corollary is a little stronger.

COROLlary 10. Let $G$ be a group such that $G / Z(G)$ is locally finite and has finite rank $r$. Then $G^{\prime}$ is locally finite and the rank of $G^{\prime}$ is $r$-bounded.

Proof. By [11, p. 102] $G^{\prime}$ is locally finite and so the above comment shows that $G^{\prime \prime}$ has finite $r$-bounded rank. Thus, we can pass to the quotient $G / G^{\prime \prime}$ and assume that $G$ is metabelian. Now Lemma 5 tells us that $r\left(G^{\prime}\right) \leq r(r-1)+r^{2}$.

We will now proceed to establish Theorem 2. First of all we note that subgroups and quotients of a generalized radical group are generalized radical as well. We also notice that periodic generalized radical groups are locally finite.

Let $G$ be a group having an ascending series whose factors are either cyclic or periodic. If the number of infinite cyclic factors in the series is finite, we call it 0-rank of $G$ and denote it by $r_{0}(G)$. If the number of infinite cyclic factors in the series is infinite, we say that $G$ has infinite 0 -rank. It is not difficult to see that the 0-rank of $G$ does not depend on the choice of the ascending series whose factors are either cyclic or periodic.

LEMMA 11. Suppose that $G$ is a finitely generated generalized radical group of finite rank $r$ and assume that $G$ has no nontrivial normal periodic subgroups. Then $G$ has a subgroup $L$ of $r$-bounded index which is soluble with $r$-bounded derived length.

Proof. It is clear that $r_{0}(A) \leq r$ for every abelian subgroup $A$ of $G$. By [3, Theorem 1] $r_{0}(G)$ is bounded in terms of $r$ only. Moreover 
[3, Theorem A] implies that $G$ has a normal series

$$
K \leq L \leq G
$$

such that $K$ is torsion-free nilpotent, $L / K$ is torsion-free abelian and $G / L$ is finite of $r$-bounded order. Since $r_{0}(G)$ is bounded in terms of $r$ only, it follows that the nilpotency class of $K$ is $r$-bounded and hence the derived length of $L$ is $r$-bounded, too.

We are now ready to prove Theorem 2 ,

Proof of Theorem 2. Recall that $G$ is a locally generalized radical group such that $G / Z(G)$ has finite rank $r$. Assume first that $G$ is finitely generated and let $T / Z(G)$ be the product of all periodic subgroups of $G / Z(G)$. Thus $T / Z(G)$ is the maximal normal periodic subgroup of $G / Z(G)$ and so $G / T$ has no nontrivial normal periodic subgroups. By Lemma $11 G$ has a subgroup $L$ of $r$-bounded index such that $L / T$ is soluble with $r$-bounded derived length. Since $G$ is a generalized radical group, it follows that all periodic sections of $G$ are locally finite and therefore so is $T / Z(G)$. Corollary 10 tells us now that $r\left(T^{\prime}\right)$ is $r$-bounded and passing to $G / T^{\prime}$ we can assume that $T^{\prime}=1$. In this case $T$ is abelian and so $L$ is soluble with $r$-bounded derived length. Lemma 5 can now be applied to deduce that $r\left(L^{\prime}\right)$ is $r$-bounded and we can pass to the quotient $G / L^{\prime}$. Now $L$ is a normal abelian subgroup and so by Lemma $4[L, G]$ has rank at most $r^{2}$. Passing to the quotient $G /[L, G]$, assume that $L$ is central. Then the index of $Z(G)$ in $G$ is $r$-bounded and by Schur's theorem the order of the derived group of $G$ is $r$-bounded as well. This proves the theorem in the case where $G$ is finitely generated. In other words, there exists an $r$-bounded number, say $R_{0}$, such that $r\left(K^{\prime}\right) \leq R_{0}$ whenever a group $K$ satisfies the hypothesis of the theorem and is finitely generated. Suppose now that our group $G$ is not necessarily finitely generated. If $r\left(G^{\prime}\right) \geq R_{0}+1$, we can choose $y_{1}, \ldots, y_{R_{0}+1} \in G^{\prime}$ such that the subgroup $\left\langle y_{1}, \ldots, y_{R_{0}+1}\right\rangle$ cannot be generated by $R_{0}$ elements. We can also choose a finitely generated subgroup $K$ in $G$ such that $y_{1}, \ldots, y_{R_{0}+1} \in K^{\prime}$. This yields a contradiction since we know that $r\left(K^{\prime}\right) \leq R_{0}$. The proof is now complete.

\section{References}

[1] S. I. Adian, On some torsion-free groups, Math. USSR-Izv., 5 (1971), 475484.

[2] J. D. Dixon, M. P. F. du Sautoy, A. Mann, D. Segal, Analytic p-adic groups, Cambridge Univ. Press, 1991. 
[3] M. R. Dixon, L. A. Kurdachenko, N. V. Polyakov, On some ranks of infinite groups, Ricerche Mat., 56 (2007), 43-59.

[4] S. Franciosi, F. de Giovanni, L. A. Kurdachenko, The Schur property and groups with uniform conjugacy classes, J. Algebra 174 (1995), 823-847.

[5] D. Gorenstein, Finite simple groups: An introduction to their classification, Plenum Press, New York and London, 1982.

[6] O. H. Kegel, B. F. A. Wehrfritz, Locally finite groups, North-Holland, Amsterdam, 1973.

[7] L. A. Kurdachenko, On groups with minimax conjugacy classes. Infinite groups and adjoining algebraic structures, Naukova Dumka Kiev, 1993, 160-177.

[8] A. Mann, The exponents of central factor and commutator groups, J. Group Theory 10 (2007), no. 4, 435-436.

[9] A. Yu. Olshanskii. Geometry of defining relations in groups. Kluwer Academic Publishers, 1991.

[10] Ya.D. Polovicky, Groups with extremal classes of conjugate elements, Sibir. Math. J. 5 (1964), 891-895.

[11] D. J. S. Robinson, Finiteness Conditions and Generalized Soluble Groups, Part 1, Springer Verlag, Berlin-New York, 1972.

[12] A. Shalev, Characterization of p-Adic Analytic Groups in Terms of Wreath Products, J. Algebra 145 (1992), 204-208.

[13] H. Zassenhaus, Beweis eines Satzes ber diskrete Gruppen, Abh. Math. Sem. Univ. Hamburg, 12 (1938), 289 - 312.

[14] E. Zelmanov, The solution of the Restricted Burnside Problem for groups of odd exponent, Math. USSR Izv., 36 (1991), 41-60.

[15] E. Zelmanov, The solution of the Restricted Burnside Problem for 2-groups, Math. Sb., 182 (1991), 568-592.

Department of Algebra, National University of Dnepropetrovsk

72 Gagarin Av., Dnepropetrovsk, Ukraine 49010

E-mail address: 1kurdachenko@i.ua

Department of Mathematics, University of Brasilia, Brasilia-DF, 70910-900 BRAZIL,PAVEL@UNB.BR

E-mail address: pavel@mat.unb.br 